\title{
Pathogenicity of SspI-positive infectious bursal disease virus and molecular characterization of the VP2 hypervariable region
}

\begin{abstract}
The pathogenicity of four isolates of infectious bursal disease virus (IBDV) that have restriction fragment length polymorphism patterns of very virulent IBDV (vvIBDV), based on the presence of SspI and TaqI sites in the VP2 hypervariable region, was studied in specific pathogen free chickens. Chickens inoculated with isolates 92/04, 94/B551 and 97/61 developed severe clinical signs with a high mortality ranging from 70 to $80 \%$, whereas the 94/273 isolate caused $10 \%$ mortality. Regardless of the isolates, significant differences were noted in the bursal lesion scores and bursa:body weight ratio index in the infected groups in comparison with the control groups. However, the presence of lesions in non-bursal tissues, muscles, thymus and at the junction of the proventriculus and gizzard were found only in the 92/04, 97/61 and 94/B551 isolates. Restriction fragment length polymorphism and sequence analysis of the VP2 hypervariable region indicated that all the isolates can be classified as vvIBDV based on the presence of SspI and TaqI sites at nucleotide positions 1011 and 833, respectively. In addition, all the isolates had amino acid substitutions at P222A, V256I and L294I, which are characteristic for vvIBDV isolated from different parts of the world. All the isolates except 94/273 also had a StyI site at nucleotide position 888 . The absence of a StyI site in this isolate was associated with amino acid substitution at 254 from $G$ to $\mathrm{S}$. The 94/273 also had an amino acid substitution at position 270 from A to E, which is variable in the STC, $\mathrm{Cu} 1$ and $\mathrm{OH}$ strains. The presence of amino acid substitutions from G254S andA270E in SspI- and TaqI-positive vvIBDV strains is very uncommon and has not been reported previously. These amino acid variations might have caused the 94/273 to become less virulent in specific pathogen free chickens and resemble a classical virulent IBDV strain.
\end{abstract}

